\title{
Audit of surgical specialty patients and beds at Pietersburg Hospital, Limpopo Province, South Africa in 2021
}

\author{
R Mavhungu, BSc; L Jonas, MB ChB, FCS (SA); A B van As, MB ChB, MMed, MBA, FCS (SA), PhD \\ Department of General Surgery, University of Limpopo, Pietersburg and Mankweng hospitals, Polokwane, South Africa
}

Corresponding author: A B van As (sebastian.vanas@uct.ac.za)

Background. While the absolute number of hospital beds is usually discussed, adequate utilisation of beds is a far better instrument to measure departmental efficiency.

Objective. To measure the number of beds for each surgical specialty in Pietersburg Hospital as well as the average length of stay (LoS) to compare bed utilisation.

Method. We conducted a 1-day descriptive cross-sectional audit of patients admitted to surgical wards on 21 April 2021 at Pietersburg Hospital.

Results. There were huge discrepancies in the number of beds per surgical specialty as well as the LoS. Over one-third of surgical beds were occupied by patients waiting for either a computed tomography scan, surgical procedure, or transfer.

Conclusion. There is a need to address the functioning of the surgical specialties with regards to the number of beds allocated as well as the ideal average length of stay.

S Afr Med J 2021;111(11b):1122-1125. https://doi.org/10.7196/SAMJ.2021.v111i11b.16124

Hospital beds are in great demand all over the world. ${ }^{[1]}$ Hospital beds are expensive; in South Africa (SA), the ballpark figure per bed per year is ZAR1 million for secondary hospitals and ZAR2 million per year for academic hospitals. Hospital managers are therefore generally pressurised to keep the total number of beds as low as possible. Although the absolute number of beds is usually discussed, the precise utilisation of the beds is often neglected. This is a huge oversight if patients are kept unnecessarily admitted, and drives the costs enormously. There are obvious competing demands for beds by the non-cutting and cutting (surgical) specialties, and decisions around this are typically political and philosophical.

According to the World Health Organization, 2 billion people around the world are estimated to have inadequate access to surgical care. ${ }^{[2]}$ Surgical care is an integral part of health systems worldwide. However, delays due to multiple transfers of surgical patients are common, even in developed countries. ${ }^{[3-5]}$ Pietersburg Hospital (PH) is a teaching hospital for the University of Limpopo, which is $31.5 \mathrm{~km}$ east of Polokwane city, the provincial capital. The hospital serves as the referral hospital for regional and district hospitals in the province. The hospital has 196 beds set aside for all adult surgical patients. Most of the beds (128) are reserved for male patients, while female patients have 68 beds. Various surgical specialties are responsible for bed allocation.

The audit reported in the present study is the first to describe the actual surgical specialty bed utilisation and the average length of stay (LoS) at a tertiary hospital in Limpopo Province, SA. In this hospital, patients are allocated to wards where beds are available, even if it is not the correct unit under which the patient should receive treatment. In an ideal situation, patients should be followed up and transferred to the correct ward as soon as a bed becomes available. If not, beds are unnecessarily occupied and this creates delay for those patients still awaiting hospital care and clinical procedures. ${ }^{[6]}$ Patients are often denied acute surgical management due to unavailability of beds.
Shortage of beds also affects the elective cases and surgical slates. If there are insufficient beds, procedures are cancelled. A shortage of intensive care unit beds alone is often the reason for cancellation of complicated surgical cases. $\mathrm{PH}$ has an ideal target of $77 \%$ bed utilisation rate and 7 days average LoS. However, the hospital consistently exceeds the bed utilisation rate target. We therefore embarked on an audit of surgical specialties with regards to the number of beds and the duration of hospital admission at $\mathrm{PH}$. We intend that the definite bed utilisation for different surgical specialty will guide the hospital management to allocate the limited available resources to service patients more efficiently.

\section{Methods}

We conducted a 1-day descriptive cross-sectional audit of patients admitted to surgical wards on 21 April 2021. All adult inpatients records were reviewed. Records belonging to a patient admitted to any of the studied surgical disciplines (general surgery, cardiothoracic, orthopaedic, neurosurgery, ear, nose and throat (ENT), maxillofacial, plastic surgery and urology) were included in the audit. Medical, gynaecological and obstetrics patients admitted to surgical wards were excluded from the audit. In addition, all children $\leq 12$ years of age were excluded for the audit. Patient files were reviewed by the research team and a data collection sheet was used to record data from the files. Ward, gender, age, surgical discipline, and diagnosis of patients were recorded. Furthermore, inpatient days before the operation, inpatient postoperative days as well as the total LoS in hospital were documented for those patients who were operated on the day of audit. The collected data were captured using Microsoft Excel (Microsoft Corp., USA). The data were analysed using STATA software (version 16; STATA Corp., USA). Continuous variables were expressed as mean and standard deviation (SD). The categorical variables were described as proportions. 


\section{Results}

A total of 142 adult surgical patients were entered into the data collection forms during the audit period. Other surgical beds were either occupied by medical or paediatric patients. The patients admitted to the surgical wards during the 1-day audit are described in Table 1. The mean age of all the patients who were admitted was 42 years. Most of the patients were males (64\%). More than one-quarter of the patients admitted to the surgical wards (27\%) required orthopaedic surgical attention. The Department of Maxillofacial Surgery had the lowest number of inpatients (0.7\%).

\section{Percentage operated}

Although the Department of Orthopaedic Surgery had the highest number of

Table 1. Descriptive summary of patients admitted to the surgical wards $(N=142)$

\begin{tabular}{|c|c|}
\hline Variables & $n(\%)^{*}$ \\
\hline \multicolumn{2}{|l|}{ Gender } \\
\hline Males & $91(64)$ \\
\hline Females & $51(36)$ \\
\hline Mean age, years & 42 \\
\hline \multicolumn{2}{|l|}{ Surgical specialty } \\
\hline Orthopaedic & $39(27.8)$ \\
\hline Neurosurgery & $30(21)$ \\
\hline General surgery & $27(19)$ \\
\hline Urology & $15(10.6)$ \\
\hline Cardiothoracic & $13(9)$ \\
\hline Plastic & $5(3.5)$ \\
\hline ENT & $4(2.8)$ \\
\hline Maxillofacial & $1(0.7)$ \\
\hline Multidisciplinary & $8(5.6)$ \\
\hline \multicolumn{2}{|l|}{ Operated patients } \\
\hline Orthopaedic & $5(11.9)$ \\
\hline Neurosurgery & $9(21.4)$ \\
\hline General surgery & $13(30.9)$ \\
\hline Urology & $4(9.5)$ \\
\hline Cardiothoracic & $2(4.8)$ \\
\hline Plastic & $2(4.8)$ \\
\hline ENT & $2(4.8)$ \\
\hline Maxillofacial & 0 \\
\hline Multidisciplinary & $5(11.9)$ \\
\hline \multicolumn{2}{|l|}{ Average LoS, days } \\
\hline Orthopaedic & 28 \\
\hline Neurosurgery & 16 \\
\hline General surgery & 7 \\
\hline Urology & 8 \\
\hline Cardiothoracic & 10 \\
\hline Plastic & 20 \\
\hline ENT & 5 \\
\hline Maxillofacial & 9 \\
\hline Multidisciplinary & 29 \\
\hline
\end{tabular}

admissions, only $11.9 \%$ of their patients underwent surgery. Almost $60 \%$ of their patients were still waiting for surgery on the day of audit (Fig. 1). We also calculated the percentage of surgical patients that were already operated on in the various surgical specialties. The Department of General Surgery had the highest number of operated patients (30\%), followed by the Department of Neurosurgery (21\%), and the departments of Plastic, Cardiothoracic and ENT conducted the same number of operations (4.8\%) (Fig.2).

\section{Average length of stay}

The average LoS differed significantly per specialty, with the shortest being 5 days for ENT, and the longest being 28 days in the Department of Orthopaedic Surgery. Multidisciplinary (complex) patients had the longest LoS (29 days) in hospital admission duration (Fig. 3). Plastic surgery and neurosurgery also had long average LoS (20 and 16 days, respectively), while general surgery, urology, cardiothoracic and maxillofacial surgery patients had average LoS between 7 - 9 days.

\section{Reasons for extended hospital stay}

We found that 46 patients were waiting for operations and half of them (50\%; $n=23$ ) were orthopaedic patients (Fig. 4). Urology, general surgery, neurosurgery, plastic surgery, ENT, and multi-disciplinary surgical patients shared the remaining $50 \%$. Seven patients were waiting for computed

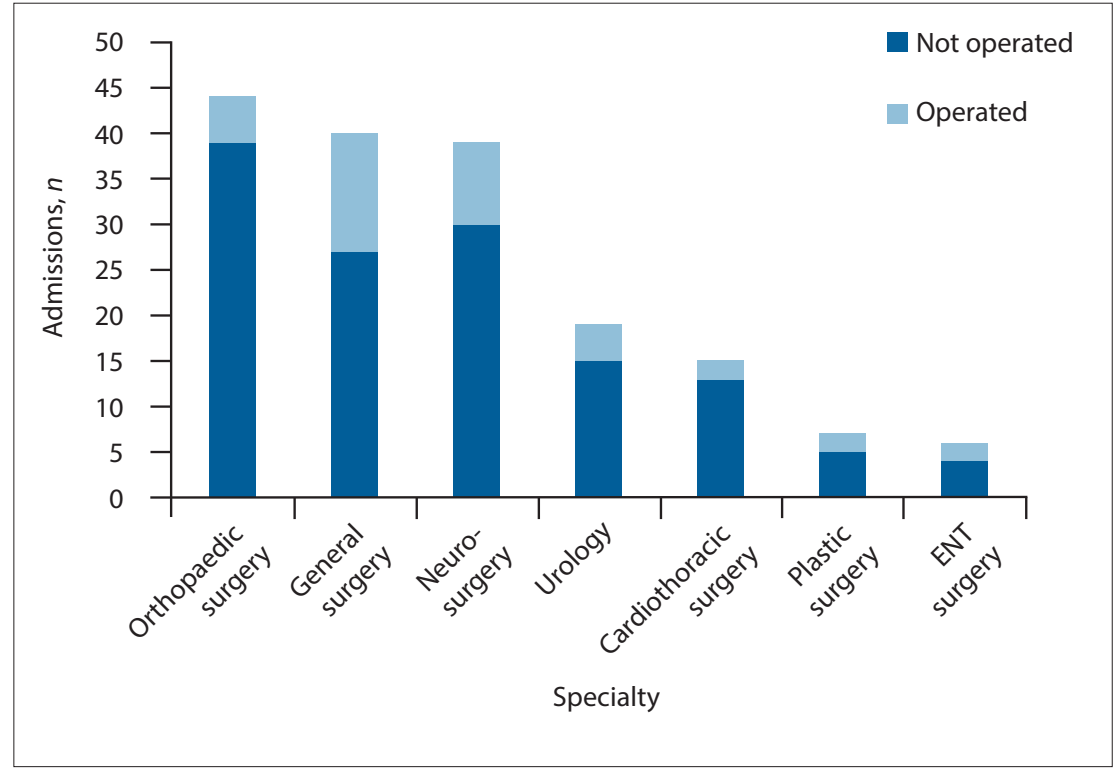

Fig. 1. Total admissions and operated patients per specialty. (ENT = ear, nose and throat.)

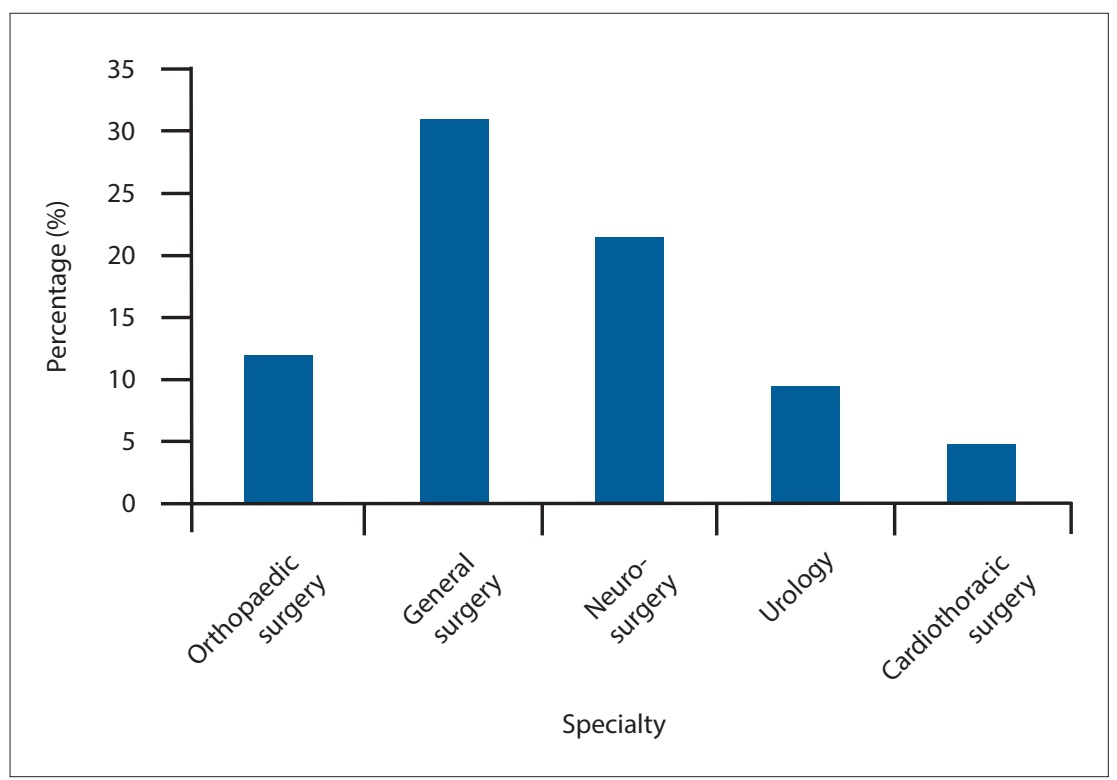

Fig. 2. Percentage of patients operated per specialty. 


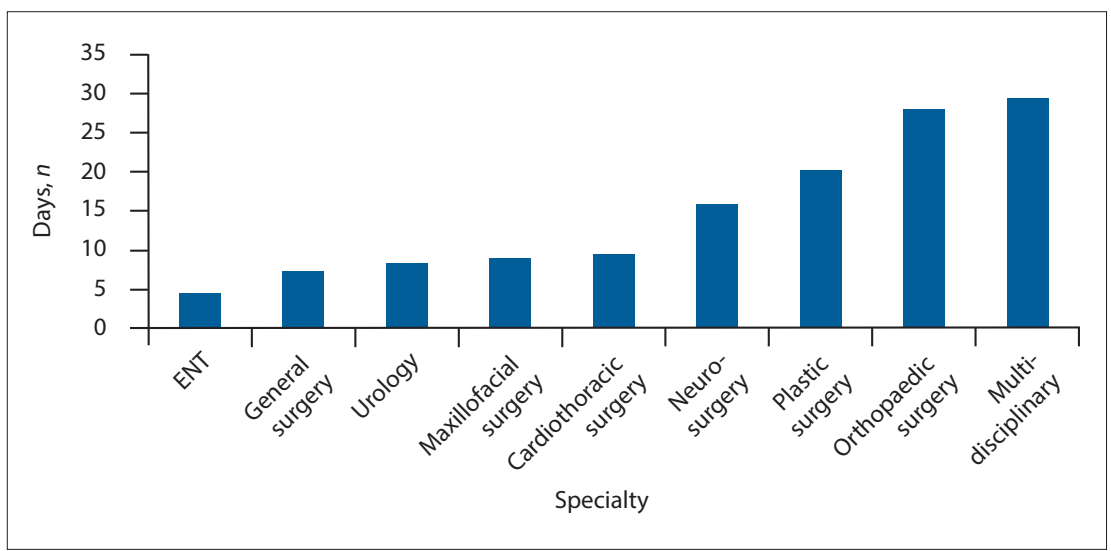

Fig. 3. Average length of stay per surgical specialty. (ENT = ear, nose and throat.)

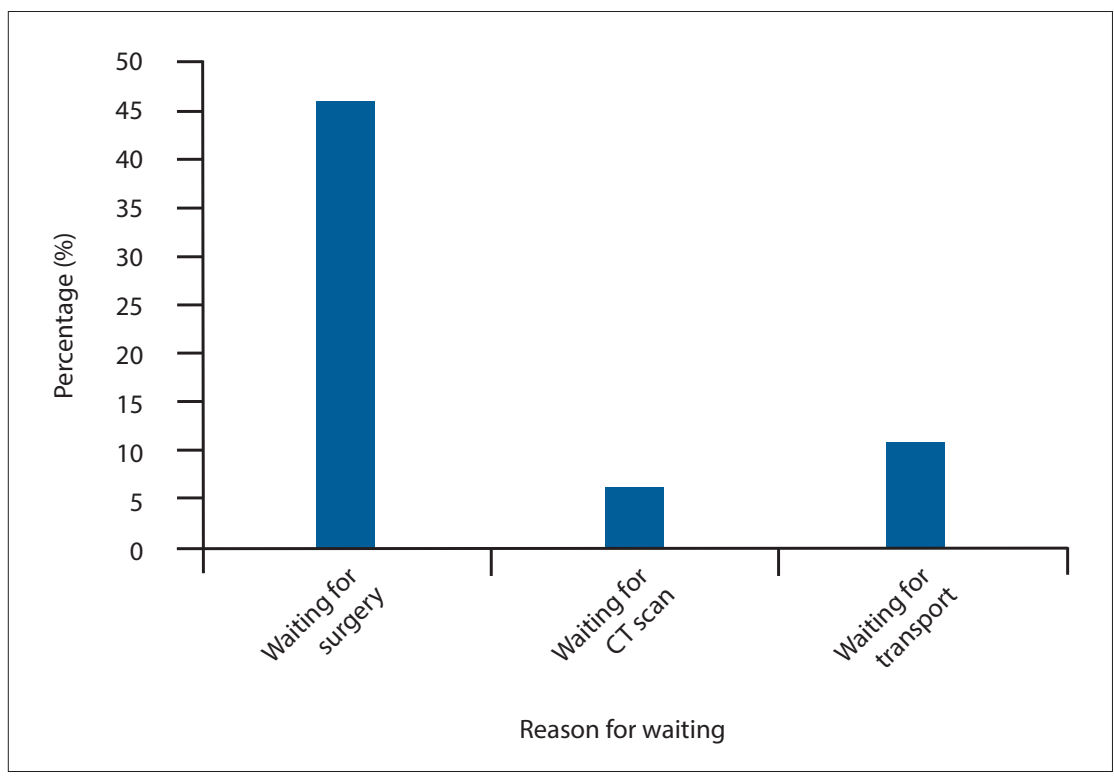

Fig. 4. Reasons for surgical patients extended hospital stay.

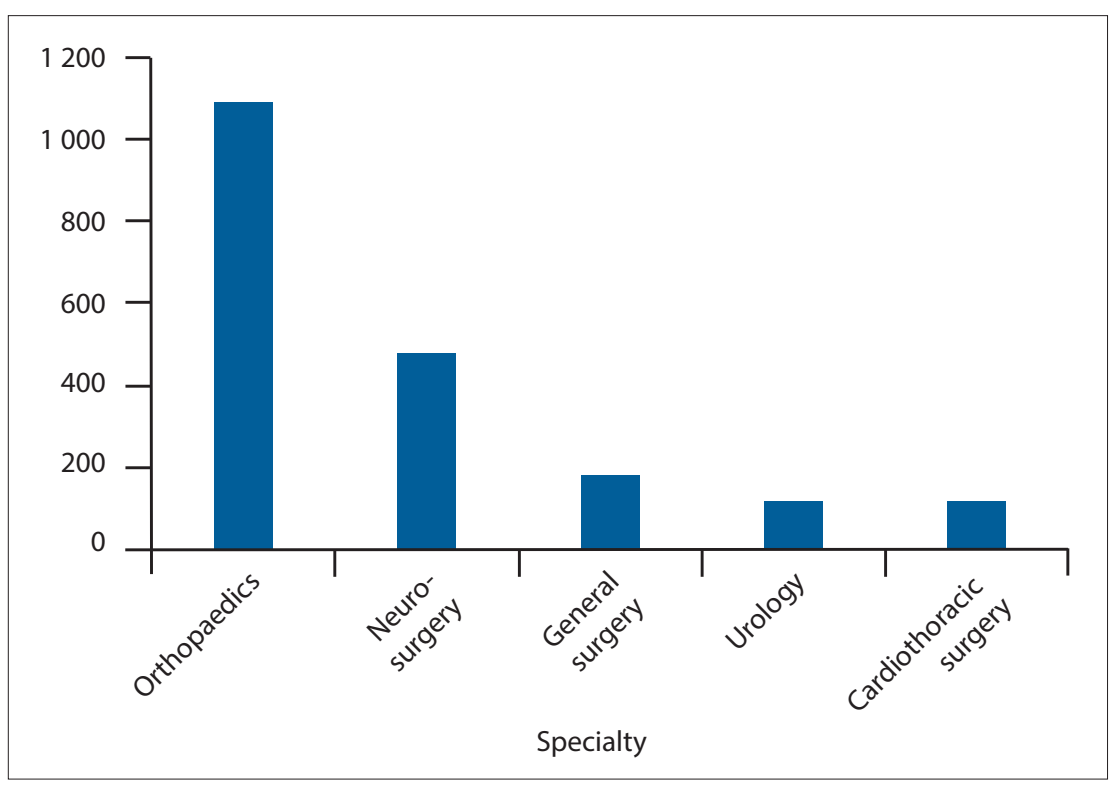

Fig. 5. Surgical admissions hospital bedload.

tomography (CT) scans, four of them were in general surgery and two in urology.
Less than a quarter (20\%) of neurosurgical admissions required rehabilitation. We also found that 11 patients were discharged but could not go home because they were waiting for transport.

\section{Hospital bedload}

We calculated the total hospital bedload of the various departments by multiplying the number of inpatients and the average LoS for the bigger surgical disciplines. The total hospital bedload of the Department of Orthopaedics was almost 6 times higher than the bedload of the Department of General Surgery (Fig. 5). The Department of Neurosurgery also had a bedload more than double that of the Department of General Surgery.

\section{Discussion}

The number of male patients was significantly higher than the number of female patients. This may reflect community disease but is more likely to reflect a gender bias in surgical admissions. Similar predisposition was reported in KwaZuluNatal, where $59.7 \%$ of the surgical load constituted male patients. ${ }^{[7]}$ There is a great disproportion in the number of bed occupancies per surgical discipline. The number of surgical beds per specialty is a function of the number of patients presenting to the specialty and the LoS. The LoS is obviously influenced by several factors such as the time duration for work-up, waiting time for investigations such as CT scanning, the availability of theatres to perform the operations; but ultimately, it is a measure of departmental efficiency. ${ }^{[8]}$ We found a substantial imbalance regarding the allocation of surgical beds to the various specialties. The greatest surgical bed occupancy was in the Department of Orthopaedic Surgery $(n=39)$. In addition, patients admitted for orthopaedic attention had the longest average $\operatorname{LoS}$ (28 days), with some staying up to 57 days without any remarkable work-up. A study in Nepal ${ }^{[9]}$ reported that an average LoS for orthopaedic inpatients at a tertiary hospital was 10.5 days. ${ }^{[9]}$ A SA report on the burden and profile of spinal pathology in Cape Town recorded a median hospital stay of 19 days. ${ }^{[10]}$ The average LoS was 7 days in the Department of General Surgery. We also found that half $(50 \%)$ of the total surgical inpatients waiting for operations were from orthopaedic surgery. General surgery $(30.9 \%)$ and neurosurgery $(21 \%)$ contributed the highest proportions of the operated patients.

The calculated total hospital bedload per surgical department indicates the significant inequity regarding hospital beds per surgical discipline, reflecting the inefficiency and 
cost of surgical beds. While these numbers may raise alarms for hospital clinical managers, we should not forget that several surgical disciplines are facing daily challenges. Not only does the number of specialists vary significantly per discipline, but the greatest challenge is the availability of theatre time. One of the greatest challenges has been the absence of a surgical emergency theatre. This has resulted in having to share the emergency theatre with the Department of Obstetrics and Gynaecology. In addition, if emergency cases fail to be operated during the night and after hours, the consequence is that the elective surgical slates are crashed, resulting in inefficient hospital bed utilisation, driving up cost and frustration for patients, doctors, and nurses.

\section{Conclusion}

Surgical bed utilisation was most ineffective for orthopaedic and neurosurgery patients. Orthopaedic patients waited for theatre space longer than all other surgical specialties. The Department of General Surgery had the average LoS that was within the prescribed hospital protocol of 7 days. Effective bed management may be improved by the appointment of a bed manager to optimise bed utilisation and minimise unnecessary admissions. Based on this audit, we further recommend performing regular 6-monthly audits on the utilisation of surgical beds and have a fixed allocation of surgical beds according to the size and theatre slate allocation of the various surgical specialties.
Declaration. None.

Acknowledgements. None.

Author contributions. Equal contributions.

Funding. None.

Conflicts of interest. None.

1. Schmidt R, Geisler S, Spreckelsen C. Decision support for hospital bed management using adaptable individual length of stay estimations and shared resources. BMC Med Inform Decis Mak 2013;13:3. https://doi.org/10.1186/1472-6947-13-3

2. Taira BR, McQueen KA, Burkle FM Jr. Burden of surgical disease: Does the literature reflect the scope of the international crisis? World J Surg 2009;33(5):893-898. https://doi.org/10.1007/s00268-009-9981-x 3. Atiyeh BS, Gunn SW, Hayek SN. Provision of essential surgery in remote and rural areas of developed as well as developing countries. Int J Surg 2010;8(8):581-585. https://doi.org/10.1016/j.ijsu.2010.07.291

4. Young JS, Bassam D, Cephas GA, et al. Inter-hospital $v$. direct scene transfer of major trauma patients in a rural trauma system. Am Surg 1998;64(1):88-91.

5. Holena DN, Mills AM, Carr BG, et al. Transfer status: A risk factor for mortality in patients with necrotising fasciitis. Surgery 2011;150(3):363-370. https://doi.org/10.1016/j.surg.2011.06.005

6. Mckenna H, Keeney S, Glenn A, Gordon P. Discharge planning: An exploratory study. J Clin Nurs 2000;9(4):594-601. https://doi.org/10.1046/j.1365-2702.2000.00384.X

. Laing GL, Skinner DL, Bruce JL, et al. Auditing surgical service provision at a South African tertiary institution: Implications for the development of district services. S Afr J surg 2017;55(4):31-35.

8. Miani C, Ball S, Pitchforth E, et al. Organisational interventions to reduce length of stay in hospital. Health Serv Deliv Res 2014;1(52). https://doi.org/10.3310/hsdr02520.

9. Mishra BN, Jha A, Maharjan E, et al. Length of stay of orthopaedic inpatients at a teaching hospital in Eastern part of Nepal. J Nepal Media Assoc 2015;53(199):182-185. https://doi.org/10.31729/jnma.2787

10. Miseer S, Mann T, Davis JH. Burden and profile of spinal pathology at a major tertiary hospital in the Western Cape, South Africa. S Afr Orthopaed J 2019;18(1). https://doi.org/10:17159/2309-8309/2019/ v18nla4

Accepted 8 September 2021. 